\title{
Transgelin 2 overexpression inhibits cervical cancer cell invasion and migration
}

\author{
QUN ZHOU, XUELU JIANG, WEI YAN and XIAOQING DOU \\ Department of Obstetrics and Gynecology, The First Affiliated Hospital of \\ Zhejiang Chinese Medical University, Hangzhou, Zhejiang 310006, P.R. China
}

Received December 1, 2017; Accepted February 5, 2019

DOI: $10.3892 / \mathrm{mmr} .2019 .10116$

\begin{abstract}
Cervical cancer is a malignancy that threatens female health. The present study aimed to investigate the role of transgelin 2 (TAGLN2) in cervical cancer. Reverse transcription-quantitative polymerase chain reaction and western blotting were conducted to detect the mRNA and protein expression levels of particular factors in HeLa cells. Cell Counting kit- 8 , wound healing and Transwell assays were conducted to determine cell viability, and migratory and invasive abilities, respectively. The results demonstrated that the expression levels of TAGLN2 were decreased in cervical cancer tissues and were associated with the survival time of patients with cervical cancer. In addition, the expression of TAGLN2 was significantly reduced in three cervical cancer cell lines (HeLa, SiHa and C-33A) compared with in a normal cervical cell line. The present study also demonstrated that TAGLN2 overexpression in HeLa cells could inhibit cell viability, migration and invasion, and it was suggested that this may occur via upregulation of the expression levels of E-cadherin and inhibitor of nuclear factor $\kappa$-light-chain-enhancer of activated $\mathrm{B}$ cells $(\mathrm{NF}-\kappa \mathrm{B})(\mathrm{I} \kappa \mathrm{B})$, and downregulation of $\mathrm{C}-\mathrm{X}-\mathrm{C}$ chemokine receptor type 4, matrix metalloproteinase (MMP)-2, MMP-9, p50 and transcription factor p65. In conclusion, TAGLN2 was revealed to inhibit cell viability, and the migratory and invasive abilities of HeLa cervical cancer cells via regulating the expression of metastasis-associated factors and the NF- $\mathrm{B}$ signaling pathway. The present study proposed a novel target gene for the diagnosis, treatment and prognosis of cervical cancer.
\end{abstract}

Correspondence to: Dr Xiaoqing Dou, Department of Obstetrics and Gynecology, The First Affiliated Hospital of Zhejiang Chinese Medical University, 54 Youdian Road, Hangzhou, Zhejiang 310006, P.R. China

E-mail: douxiaoqing@126.com

Key words: transgelin 2, cervical cancer, cell invasion, metastasis, nuclear factor $\kappa$-light-chain-enhancer of activated B cells

\section{Introduction}

Cervical cancer is the fourth most common type of cancer diagnosed among women worldwide (1). A combined treatment regimen of screening, surgery and radiotherapy has improved the prognosis of early stage cervical cancer; however, it remains difficult to prevent metastasis and to treat recurring cancer at the advanced stages, which are the main causes of mortality in patients with cervical cancer (2). The prominent cause of cervical cancer has been reported to be human papillomavirus (HPV) infection; the most prevalent carcinogenic HPV strains are HPV-16 and HPV-18 (3). The present study aimed to investigate the molecular mechanism underlying the invasion and metastasis of cervical cancer, which may aid developments in its prognosis and improve the curative rate of patients with cervical cancer.

Nuclear factor $\kappa$-light-chain-enhancer of activated B cells $(\mathrm{NF}-\kappa \mathrm{B})$ is a protein complex that controls DNA transcription and affects cell survival. All proteins in the NF- $\mathrm{B}$ family contain an N-terminal Rel homology domain (RHD), which possesses a nuclear-localization sequence (NLS), and some RHDs also contain an additional C-terminal transactivation domain (4). NF- $\kappa \mathrm{B}$ signaling serves important roles in transmitting signals from the cell membrane to the nucleus. As a transcription factor, $\mathrm{NF}-\kappa \mathrm{B}$ can be rapidly activated and responds to stimuli in the early stage of cervical cancer, including stress, cytokines, and bacterial and viral antigens (5). Notably, the abnormal regulation of NF- $\kappa \mathrm{B}$ has been reported to lead to improper development of the immune system, the development of inflammatory and autoimmune diseases, and cancer $(6,7)$.

Transgelin 2 (TAGLN2) is a member of the actin- and calmodulin-binding protein family $(8,9)$, which regulates cell morphology and motility (10). TAGLN2 has been proposed as a tumor suppressor in cancer metastasis (11). The TAGLN family has been reported to interfere in extracellular signal-regulated kinase (ERK)1 and ERK2 signaling, which is dependent on the calponin protein domain of the TAGLN family (12). Additionally, members of the TAGLN family can decrease the expression of matrix metalloproteinase (MMP)-9 to inhibit cancer metastasis (13); however, research has suggested that TAGLN2 serves as an oncogene in numerous types of cancer, including head and neck squamous cell carcinoma (14), renal cell carcinoma (15) and bladder cancer (16). Therefore, the 
function of TAGLN2 is controversial and it is of importance to investigate the role of TAGLN2 in cervical cancer.

The present study aimed to determine the role of TAGLN2 in cervical cancer and its underlying mechanism. The findings of the present study may not only improve current understanding of the progression of cervical cancer, but may lead to the identification of a potential therapeutic target for the treatment of cervical cancer.

\section{Materials and methods}

Patients and tissues. Human cervical tissues were collected from 56 female patients with cervical cancer (age, 36-71 years) and 56 female patients without cervical cancer who underwent hysterectomy for uterine fibroids (normal cervical tissue, control) at The First Affiliated Hospital of Zhejiang Chinese Medical University (Hangzhou, China) between June 2015 and June 2017. According to the International Federation of Gynecology and Obstetrics (FIGO) criteria (17), 25 of the 56 patients with cervical cancer were of the I/II stages, whereas the remaining 31 cases were of the III/IV stages. None of the 56 patients with cervical cancer received radiotherapy or chemotherapy prior to surgery. All sample collections were approved by an institutional review board of the Ethics Committee of The First Affiliated Hospital of Zhejiang Chinese Medical University, and informed consent was obtained from each patient. The tissues samples were preserved in liquid nitrogen for reverse transcription-quantitative polymerase chain reaction (RT-qPCR) and western blot analysis. Patients were divided into higher and lower TAGLN2 expression groups, using mean TAGLN2 expression as the cut-off value.

Cell culture. Human cervical cancer cell lines, including HeLa (HPV-18-positive), SiHa (HPV-16-positive) and C-33A (HPV-negative), were purchased from the Shanghai Institute of Cell Biology, Chinese Academy of Sciences (Shanghai, China). Human normal cervical epithelial cells (HcerEpic) (cat. no. BNCC340374) were purchased from Bena Culture Collection (Beijing, China). Cells were cultured in high-glucose Dulbecco's modified Eagle's medium (Gibco; Thermo Fisher Scientific, Inc., Waltham, MA, USA) supplemented with $10 \%$ fetal bovine serum (FBS; Gibco; Thermo Fisher Scientific, Inc.) and $1 \%$ penicillin-streptomycin (Gibco; Thermo Fisher Scientific, Inc.) in a cell incubator containing $5 \% \mathrm{CO}_{2}$ and $50 \%$ humidity at $37^{\circ} \mathrm{C}$. The medium was replaced with fresh cell culture medium every 2 days and cells in the logarithmic phase were employed for subsequent analysis. RT-qPCR and western blotting were conducted to detect and compare the expression levels of TAGLN2 among the various cell lines.

Cell transfection. The TAGLN2 sequence was inserted into a pcDNA3.1-Flag plasmid (Invitrogen; Thermo Fisher Scientific, Inc.). HeLa cells were seeded in 12-well plates at an initial concentration of $6 \times 10^{4}$ cells/well. The following day, cells were transfected with $1 \mu \mathrm{g}$ TAGLN2 overexpression recombinant plasmid or $1 \mu \mathrm{g}$ empty plasmid vector [overexpression and negative control (NC) groups, respectively] using the transfection reagent Lipofectamine ${ }^{\circledR} 2000$ (Invitrogen; Thermo Fisher Scientific, Inc.) at $37^{\circ} \mathrm{C}$ for $6 \mathrm{~h}$. Untreated cells were considered the control group. Prior to conducting functional analyses, cells were cultured in normal complete medium for $48 \mathrm{~h}$ post-transfection. Cell transfection efficiency was assessed by analyzing the expression levels of TAGLN2 via RT-qPCR and western blotting.

Cell viability assay. Cell Counting kit-8 (CCK-8; Beyotime Institute of Biotechnology, Nantong, China) was used to investigate cell viability post-transfection. Cells were seeded in 96-well plates at an initial density of $5 \times 10^{3}$ cells/well and were incubated for 12,24 and $48 \mathrm{~h}$ at $37^{\circ} \mathrm{C}$. After incubating with $20 \mu \mathrm{l} \mathrm{CCK}-8$ reagent for $1 \mathrm{~h}$ at $37^{\circ} \mathrm{C}$, a microplate reader was used for analysis (BioTek Instruments, Inc., Winooski, VT, USA). Optical density was measured at $450 \mathrm{~nm}$.

Wound healing assay. The cell migratory abilities of the TAGLN2 overexpression group were determined using a wound healing assay, and were compared with the abilities of the control and NC groups. Cells were seeded in 12-well plates at an initial concentration of $1 \times 10^{5}$ cells/well. Following incubation at $37^{\circ} \mathrm{C}$ for $24 \mathrm{~h}$, the confluent monolayer cells were scratched gently using a $200-\mu 1$ pipette tip to obtain a cell-free area. Subsequently, cells were cultured for a further $24 \mathrm{~h}$ at $37^{\circ} \mathrm{C}$. Finally, the area of the cell-free zone was observed under an optical microscope (Olympus Corporation, Tokyo, Japan) and the rate of cell migration was calculated relative to the control group.

Cell invasion assay. The cell invasive abilities of the TAGLN2 overexpression group were examined and compared with the abilities of the control and NC groups via a Transwell invasion assay. Specifically, each Transwell chamber (Corning Incorporation, Corning, NY, USA) was coated with $100 \mu \mathrm{l}$ melted Matrigel (BD Biosciences, Franklin Lakes, NJ, USA) and the chambers were maintained for $30 \mathrm{~min}$ at $37^{\circ} \mathrm{C}$ to ensure solidification of the Matrigel. Subsequently, $100 \mu 1$ serum-free medium-diluted cell suspensions $\left(1 \times 10^{6} / \mathrm{ml}\right)$ were added into the upper chambers, whereas $600 \mu 1$ complete culture medium containing $10 \%$ FBS was added to the bottom chambers. Following incubation for $24 \mathrm{~h}$ at $37^{\circ} \mathrm{C}$, the invaded cells were fixed with methanol for $15 \mathrm{~min}$ at room temperature and were stained with $0.1 \%$ crystal violet for $30 \mathrm{~min}$ at room temperature. Subsequently, five random high-power fields were observed under an optical microscope (Olympus Corporation). The rate of cell invasion was calculated relative to the control group.

$R T$ - $q P C R$. mRNA expression levels were determined via RT-qPCR analysis. Total RNA was extracted from frozen tissues or the various cell groups using TRIzol ${ }^{\circledR}$ reagent (Invitrogen; Thermo Fisher Scientific, Inc.). cDNA was reverse transcribed from $1 \mu \mathrm{g}$ RNA using PrimeScript RT Master Mix (Takara Biotechnology Co., Ltd., Dalian, Japan), according to the manufacturer's protocol. The primer sequences for TAGLN2, E-cadherin, C-X-C chemokine receptor type 4 (CXCR4), MMP-2, MMP-9, p50, transcription factor p65 (RelA), inhibitor of NF- $\kappa \mathrm{B}(\mathrm{I} \kappa \mathrm{B})$ and $\beta$-actin were designed and synthesized by Invitrogen (Thermo Fisher Scientific, Inc.); the sequences are presented in Table I. $\beta$-actin served as an internal control. qPCR amplification was then conducted 
Table I. Primer sequences applied in the present study.

\begin{tabular}{|c|c|c|}
\hline Gene & Direction & Sequence $\left(5^{\prime}-3^{\prime}\right)$ \\
\hline \multirow[t]{2}{*}{$\beta$-actin } & Forward & GTGGACATCCGCAAAGAC \\
\hline & Reverse & GAAAGGGTGTAACGCAACT \\
\hline \multirow[t]{2}{*}{ TAGLN2 } & Forward & AGTGACATTCCCAGAGAGCC \\
\hline & Reverse & GGCCCCTAAATTTTGGTCCC \\
\hline \multirow[t]{2}{*}{ E-cadherin } & Forward & ACGCATTGCCACATACACTC \\
\hline & Reverse & GGTGTTCACATCATCGTCCG \\
\hline \multirow[t]{2}{*}{ MMP-2 } & Forward & CAGCCCTGCAAGTTTCCATT \\
\hline & Reverse & GTTGCCCAGGAAAGTGAAGG \\
\hline \multirow[t]{2}{*}{ MMP-9 } & Forward & GAGACTCTACACCCAGGACG \\
\hline & Reverse & GAAAGTGAAGGGGAAGACGC \\
\hline \multirow[t]{2}{*}{ CXCR4 } & Forward & TGTCATCACGCTTCCCTTCT \\
\hline & Reverse & TTCCTTGGCCTCTGACTGT \\
\hline \multirow[t]{2}{*}{$\mathrm{p} 50$} & Forward & GGTGACAGGAGACGTGAAGA \\
\hline & Reverse & TCCACCACATCTTCCTGCTT \\
\hline \multirow[t]{2}{*}{ RelA } & Forward & CTACACAGGACCAGGGACAG \\
\hline & Reverse & GGAAGGGGTTGTTGTTGGTC \\
\hline \multirow[t]{2}{*}{$\mathrm{I} \kappa \mathrm{B}$} & Forward & TATCACGGAGACCCAGGAGA \\
\hline & Reverse & GCTTGTGAATCTGCTCCTCG \\
\hline
\end{tabular}

CXCR4, C-X-C chemokine receptor type 4; I $\mathrm{B}$, inhibitor of nuclear factor $\kappa$-light-chain-enhancer of activated $\mathrm{B}$ cells; MMP, matrix metalloproteinase; RelA, transcription factor p65.

using SYBR Premix Ex Taq (Takara Biotechnology Co., Ltd.) on an ABI 7500 thermocycler system (Applied Biosystems; Thermo Fisher Scientific, Inc.), under the following conditions: Pre-denaturation at $95^{\circ} \mathrm{C}$ for $30 \mathrm{sec}$, followed by 40 cycles of denaturation at $95^{\circ} \mathrm{C}$ for $5 \mathrm{sec}$, and annealing/extension at $60^{\circ} \mathrm{C}$ for $35 \mathrm{sec}$, and a final step at $95^{\circ} \mathrm{C}$ for $15 \mathrm{~min}$. Data were analyzed using the 7500 Fast System Sequence Detection Software v1.3.1 (Applied Biosystems; Thermo Fisher Scientific, Inc.). The relative gene expression levels were calculated using the $2^{-\Delta \Delta \mathrm{Cq}}$ method (18).

Western blotting. Protein was extracted from frozen tissues and cells using the Tissue or Cell Total Protein Extraction kit (Sangon Biotech Co., Ltd., Shanghai, China). Total protein concentration was determined using a Bicinchoninic Acid assay (Beyotime Institute of Biotechnology). Proteins $(20 \mu \mathrm{g})$ were then separated by $10 \%$ SDS-PAGE and were then transferred to polyvinylidene fluoride (PVDF) membranes, which were used to conduct immunoblotting. The PVDF membranes were blocked with $5 \%$ nonfat milk for $1 \mathrm{~h}$ at room temperature. Subsequently, PVDF membranes were probed with specific primary antibodies at $4^{\circ} \mathrm{C}$ overnight, and were then incubated with appropriate horseradish peroxidase-conjugated secondary antibodies (goat anti-rabbit; 1:2,000; cat. no. ab205718 and goat anti-mouse; 1:5,000; cat. no. ab205719; both Abcam, Cambridge, MA, USA) at $37^{\circ} \mathrm{C}$ for $1 \mathrm{~h}$. Subsequently, proteins were exposed to X-ray film, and immunoreactive bands were detected using enhanced chemiluminescence detection reagents (GE Healthcare, Chicago, IL, USA). $\beta$-actin was used as a loading control. Protein quantities were determined by Lab Works Image Acquisition and Analysis Software 4.0 (UVP, LLC, Phoenix, AZ, USA). The primary antibodies employed were as follows: Rabbit anti-TAGLN2 (1:500, cat. no. sc-166697; Santa Cruz Biotechnology, Inc., Dallas, TX, USA), anti-E-cadherin (1:1,000, cat. no. ab133597; Abcam), anti-CXCR4 (1:1,000, cat. no. ab181020; Abcam), anti-MMP-2 (1:1,000, cat. no. ab92536; Abcam), anti-MMP-9 (1:1,000, cat. no. ab38898; Abcam), anti-p50 (1:1,000, cat. no. ab32360; Abcam), anti-RelA (1:1,000, cat. no. ab16502; Abcam), anti-IкB (1:1,000, cat. no. ab32518; Abcam) and anti- $\beta$-actin (1:2,000, cat. no. ab8227; Abcam).

Statistical analysis. Statistical analysis was conducted using SPSS 22.0 (IBM Corp., Armonk, NY, USA). Each experiment was repeated three times. Data are presented as the means \pm standard deviation. One-way analysis of variance followed by Tukey's test was used to calculate statistical significance. A $\chi^{2}$ test was used to analyze categorical variables. Survival was investigated via Kaplan-Meier analysis and log-rank test. $\mathrm{P}<0.05$ was considered to indicate a statistically significant difference. $\mathrm{P}<0.01$ was considered to indicate a highly statistically significant difference.

\section{Results}

Expression levels of TAGLN2 are associated with the severity of cervical carcinoma. To understand the biological function of TAGLN2 in cervical cancer, the expression levels of TAGLN2 in cervical cancer tissues were determined by RT-qPCR and western blotting. The results revealed that the mRNA expression levels of TAGLN2 were significantly lower in cervical cancer tissues than in normal cervical tissues (Fig. 1A; $\mathrm{P}<0.05$ ). The representative western blot bands are presented in Fig. 1B; the protein expression levels of TAGLN2 were significantly lower in cervical cancer tissues compared with in normal tissues (Fig. 1C). The clinical pathological features were also evaluated, which indicated that independent of age, the expression levels of TAGLN2 were associated with FIGO stages and the histological grades of cervical cancer, and the advanced stages of cancer were associated with higher incidences of low TAGLN2 expression (Table II). Survival analysis demonstrated that patients with cervical cancer and higher expression levels of TAGLN2 survived for longer than those with lower levels of TAGLN2 (Fig. 1D; P<0.05).

Effects of TAGLN2 on the viability of cervical cancer cells. The expression levels of TAGLN2 were determined in three cervical cancer cell lines, including HeLa (HPV-18-positive), SiHa (HPV-16-positive) and C-33A (HPV-negative). The results demonstrated that the expression levels of TAGLN2 were significantly lower in HeLa and C-33A cells, and were markedly decreased in $\mathrm{SiHa}$ cells compared with in normal cervical cells (Fig. 2A and B; $\mathrm{P}<0.05$ ). To investigate the function of TAGLN2 in cervical cancer, a TAGLN2 overexpression model was generated using HeLa cells, which exhibited the lowest levels of TAGLN2 expression compared with in $\mathrm{SiHa}$ and $\mathrm{C}-33 \mathrm{~A}$ cells.

The mRNA and protein expression levels of TAGLN2 were determined following the overexpression of TAGLN2. The data revealed significantly higher expression levels of 
A

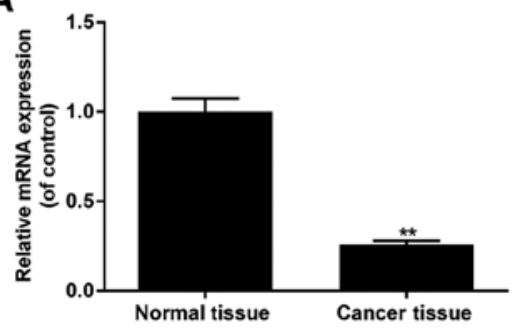

B

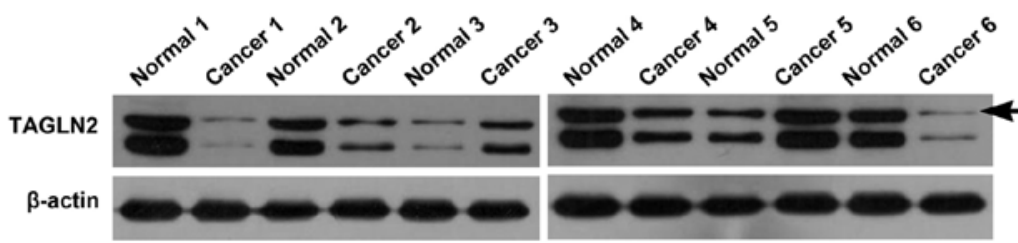

C

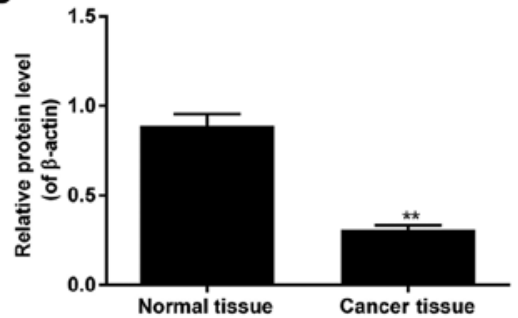

D

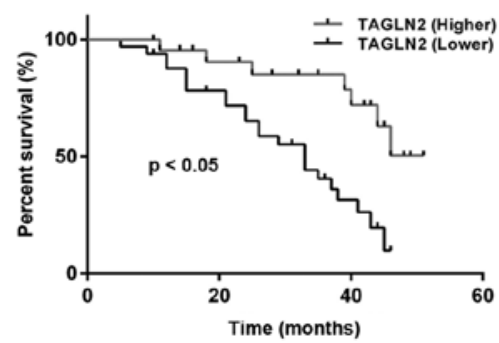

Figure 1. Expression levels of TAGLN2 in cervical carcinoma. (A) mRNA expression levels of TAGLN2 in 56 cervical cancer tissues and corresponding normal tissues, as determined by reverse transcription-quantitative polymerase chain reaction. (B and C) Protein expression levels of TAGLN2 in randomly selected patients, as determined by western blotting. The arrow indicates the main protein band for TAGLN2, which was used to calculate the relative protein expression level. (D) Survival analysis demonstrated that patients with cervical cancer and high expression levels of TAGLN2 exhibited longer survival than patients with lower levels. ${ }^{* *} \mathrm{P}<0.01$ vs. normal tissues. TAGLN2, transgelin 2.

A

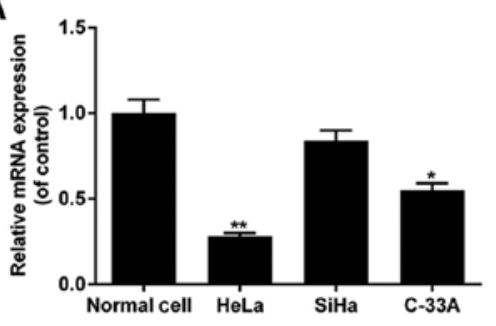

C

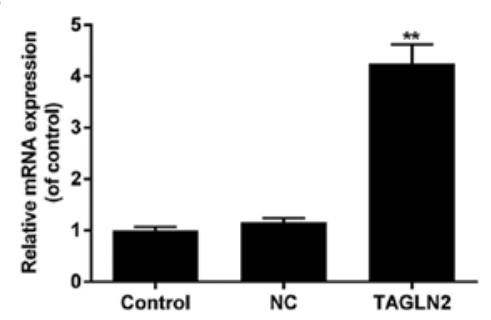

B
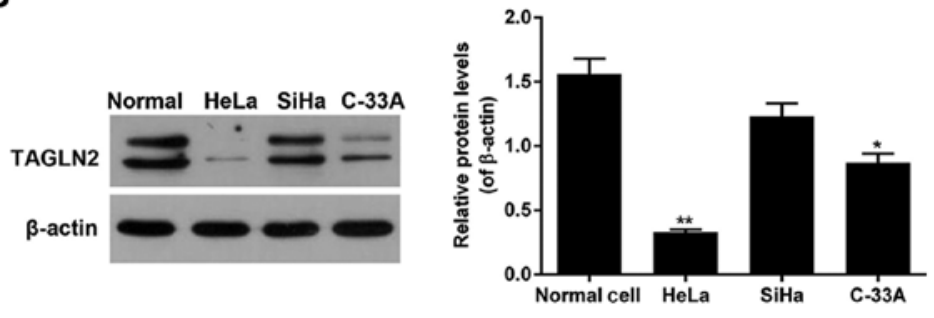

D

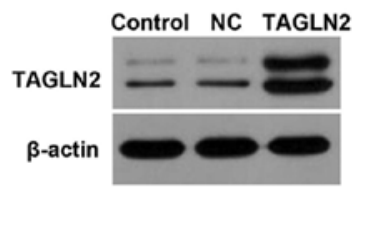

\section{E}

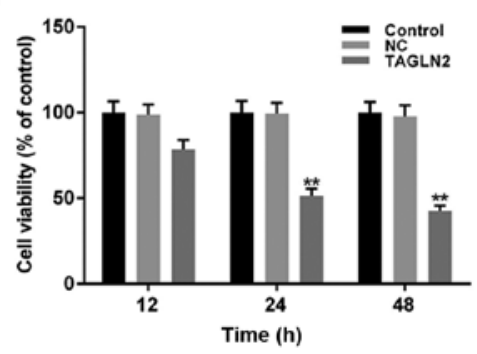

Figure 2. TAGLN2 overexpression inhibits the viability of cervical cancer cells. (A) mRNA and (B) protein expression levels of TAGLN2 were detected in HeLa, SiHa and C-33A cervical cancer cell lines via reverse transcription-quantitative polymerase chain reaction and western blotting, respectively. ${ }^{*} \mathrm{P}<0.05$, ${ }^{* *} \mathrm{P}<0.01$ vs. Normal cell group. (C) mRNA and (D) protein expression levels of TAGLN2 were determined following TAGLN2 transfection. Significantly higher expression levels of TAGLN2 were detected in the TAGLN2 overexpression group compared with in the NC and control groups. (E) Cell viability of the TAGLN2 group was evaluated using a Cell Counting Kit-8 assay, which revealed that cell viability was inhibited in a time-dependent manner (12, 24 and $48 \mathrm{~h}$ ), compared with in the NC and control groups. ${ }^{* *} \mathrm{P}<0.01$ vs. Control group. NC, negative control; TAGLN2, transgelin 2. 
Table II. Analysis of TAGLN2 expression in cervical carcinoma.

TAGLN2 expression levels

\begin{tabular}{lcrrr}
\cline { 3 - 5 } Characteristic & Factor $(\mathrm{n})$ & Low & High & P-value \\
\hline Age (years) & $<50$ & 16 & 12 & 0.786 \\
& I & 17 & 11 & 19 \\
FIGO stages & I and II (25) & 27 & 4 & $0.001^{\mathrm{a}}$ \\
& Low differentiation & 9 & 16 & $0.002^{\mathrm{a}}$ \\
Histological grade & High differentiation & 24 & 7 &
\end{tabular}

${ }^{\mathrm{a}} \mathrm{P}<0.05, \chi^{2}$ test. TAGLN2, transgelin 2 .

A
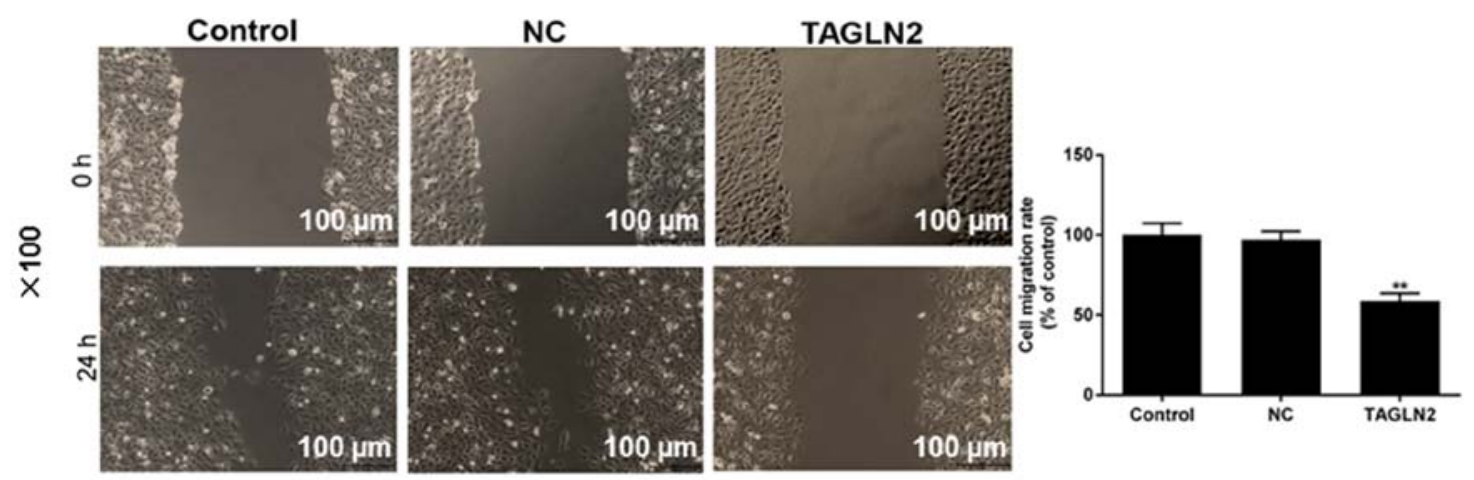

B
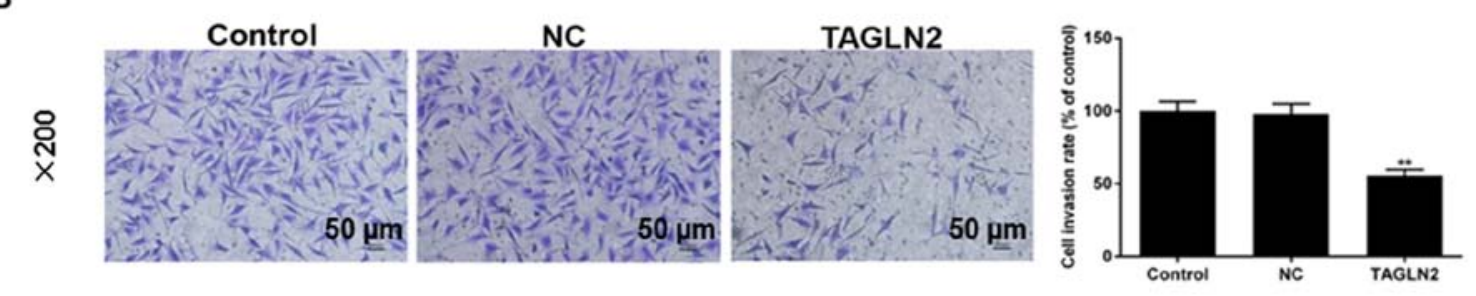

C

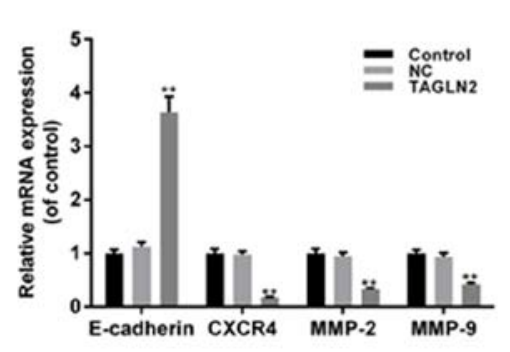

D

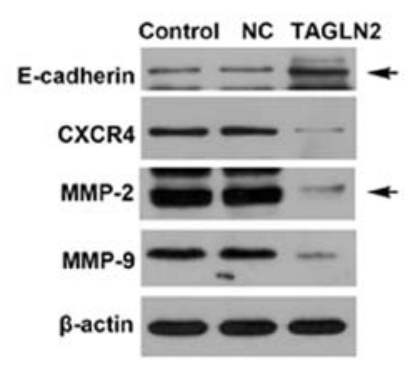

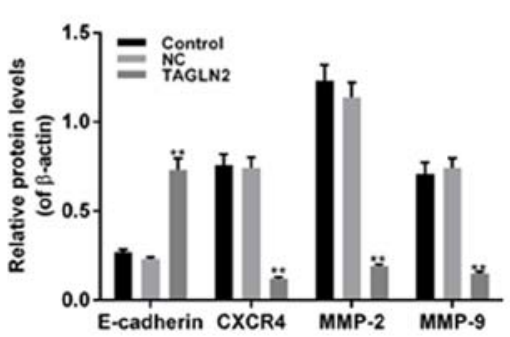

Figure 3. TAGLN2 overexpression inhibits the migratory and invasive abilities of cervical cancer cells. (A) The migratory ability of HeLa cells was measured following TAGLN2 transfection by wound healing assay (magnification, x100). (B) The invasive ability of HeLa cells was measured following TAGLN2 transfection by Transwell assay (magnification, x200). The expression levels of migration- and invasion-associated factors were examined by (C) reverse transcription-quantitative polymerase chain reaction and (D) western blotting. The arrows indicate the main protein bands. ${ }^{* *} \mathrm{P}<0.01 \mathrm{vs}$. the NC and control groups. CXCR4, C-X-C chemokine receptor type 4; MMP, matrix metalloproteinase; NC, negative control; TAGLN2, transgelin 2.

TAGLN2 in the TAGLN2 overexpression group compared with in the $\mathrm{NC}$ and control groups (Fig. $2 \mathrm{C}$ and $\mathrm{D} ; \mathrm{P}<0.05$ ). The cell viability of the TAGLN2 overexpression group was evaluated using a CCK-8 assay; TAGLN2 was revealed to significantly inhibit cell viability in a time-dependent manner (12, 24 and $48 \mathrm{~h})$, compared with in the NC and control groups (Fig. 2E; P<0.05).
Effects of TAGLN2 on the migratory and invasive abilities of cervical cancer cells. To further investigate the biological function of TAGLN2 in cervical cancer cells, cell migratory and invasive abilities were analyzed following TAGLN2 overexpression via wound healing and Transwell invasion assays, respectively. The results demonstrated that cell migration and invasion were significantly suppressed in the TAGLN2 
A

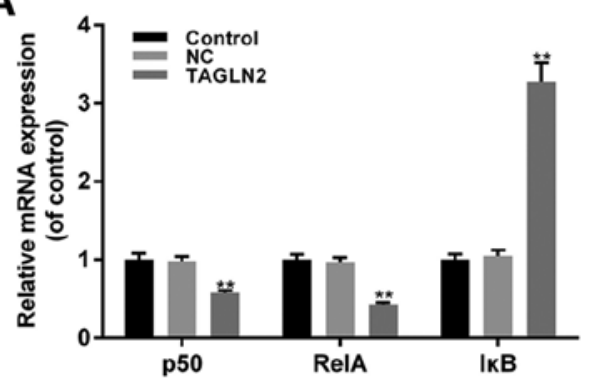

B

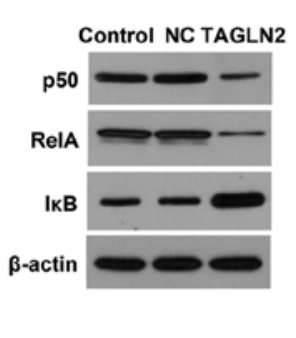

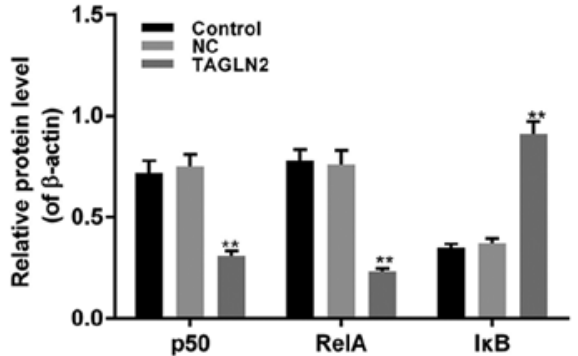

Figure 4. TAGLN2 overexpression inhibits the NF- $\mathrm{B}$ signaling pathway in cervical cancer cells. (A) mRNA and (B) protein expression levels of the main factors associated with the $\mathrm{NF}-\kappa \mathrm{B}$ signaling pathway were examined by reverse transcription-quantitative polymerase chain reaction and western blotting, respectively. ${ }^{* *} \mathrm{P}<0.01$ vs. the $\mathrm{NC}$ group. I $\mathrm{B}$, inhibitor of $\mathrm{NF}-\kappa \mathrm{B} ; \mathrm{NC}$, negative control; NF- $\mathrm{B}$, nuclear factor $\kappa$-light-chain-enhancer of activated $\mathrm{B}$ cells; RelA, transcription factor p65; TAGLN2, transgelin 2.

overexpression group compared with in the $\mathrm{NC}$ and control groups (Fig. 3A and $\mathrm{B} ; \mathrm{P}<0.05$ ). In addition, $\mathrm{RT}-\mathrm{qPCR}$ and western blotting demonstrated that the mRNA and protein expression levels of E-cadherin were significantly increased, whereas those of CXCR4, MMP-2 and MMP-9 were significantly decreased in the TAGLN2 overexpression group compared with in the $\mathrm{NC}$ and control groups (Fig. 3C and D; $\mathrm{P}<0.05)$. These findings indicated that TAGLN2 overexpression may inhibit cell migratory and invasive abilities via regulating the expression of associated factors in HeLa cells.

Effects of TAGLN2 on the $N F-\kappa B$ signaling pathway in cervical cancer cells. In addition to migration- and invasion-associated factors, the function of TAGLN2 in the NF- $\kappa \mathrm{B}$ signaling pathway was investigated. The results demonstrated that the expression levels of p50 and RelA were significantly decreased, whereas those of I $\mathrm{B}$ were significantly increased in the TAGLN2 overexpression group compared with in the $\mathrm{NC}$ and control groups (Fig. $4 \mathrm{~A}$ and $\mathrm{B} ; \mathrm{P}<0.05$ ). These findings suggested that the inhibitory effects of TAGLN2 on cell metastasis and invasion of HeLa cervical cancer cells may be associated with the expression of factors associated with the $\mathrm{NF}-\kappa \mathrm{B}$ signaling pathway.

\section{Discussion}

Cervical cancer is a malignancy that threatens female health. The main issue affecting cervical cancer treatment is that it is difficult to treat metastasizing or recurring cervical cancer in patients in the advanced stages $(19,20)$. As a tumor suppressor associated with cancer metastasis (11), the present study proposed that TAGLN2 may exert tumor-suppressing functions in cervical cancer. A total of 56 cervical cancer tissue samples, as well as 56 corresponding normal tissues from healthy patients were collected in the present study. In addition, HeLa cervical cancer cells were employed to successfully construct a TAGLN2 overexpression cell model to determine the function of TAGLN2 and its underlying molecular mechanism.

In the present study, the expression levels of TAGLN2 were decreased in cervical cancer tissues, and the expression of TAGLN2 was associated with the clinical features of cervical cancer. Higher degrees of differentiation and FIGO grades of cervical cancer were associated with low expression levels of TAGLN2, which were independent of age. Additionally, patients with cervical cancer and higher levels of TAGLN2 expression exhibited longer periods of survival than those with lower TAGLN2 expression levels. These findings indicated that TAGLN2 may act as a suppressing factor in cervical cancer.

It has been reported that HPV infection, particularly with HPV-16 or HPV-18, is a prominent cause of cervical cancer (21). Therefore, three representative cervical cancer cell lines: HeLa (HPV-18-positive), SiHa (HPV-16-positive) and C-33A cells (HPV-negative), were employed to determine the expression levels of TAGLN2 in the present study. It was revealed that the expression of TAGLN2 was decreased in these three cell lines, and that the expression of TAGLN2 was markedly lower in HeLa and C-33A cells than in SiHa cells. Therefore, the expression of TAGLN2 may be partially independent of HPV infection; however, further investigation is required. To determine the biological mechanism underlying the suppressive effects of TAGLN2 on cervical cancer, a TAGLN2 overexpression model was generated in HeLa cells to evaluate the function of TAGLN2 in cervical cell viability, and migration and invasion. The results of the present study revealed that overexpression of TAGLN2 may inhibit the viability of HeLa cells, and cell migratory and invasive abilities were also suppressed by TAGLN2. Notably, the reduced migration and invasion of HeLa cells may be partly explained by reduced cell viability.

Epithelial-mesenchymal transition (EMT) is a critical mechanism of metastasis and invasion in epithelial cells $(22,23)$. During EMT, cells lose polarity and the expression of E-cadherin is reduced. MMPs belong to the family of calcium-dependent zinc-containing endopeptidases, which serve roles in degradation of the extracellular matrix, and induce tumor metastasis and invasion (24,25). MMP-2 and MMP-9 can induce the degradation of basement membranes by degrading the main component, type IV collagen, facilitating cell invasion and tumor metastasis (26). CXCR4 serves critical functions in the metastasis and invasion of malignant tumors, and is involved in the process of embryo generation $(27,28)$. A previous study reported that overexpression of TAGLN2 inhibits the expression of MMP-9 and suppresses fibrosarcoma cell invasion (13). These observations are consistent with those of the present study, in which overexpression of TAGLN2 was associated with the inhibited migration and invasion of cervical cancer cells. In addition, overexpression of TAGLN2 was suggested to promote the expression of 
E-cadherin, whereas the expression levels of MMP-2, MMP-9 and CXCR4 were reduced.

The NF- $\kappa$ B signaling pathway is critical for the regulation of tumor proliferation and metastasis $(29,30)$. Under normal conditions, I $\mathrm{B}$ binds to $\mathrm{NF}-\kappa \mathrm{B}$ via its $\mathrm{C}$-terminal specific ankyrin repeat motif to obstruct the NLS of NF- $\kappa$ B and suppress its nuclear translocation (31). Classical activation of the NF- $\kappa \mathrm{B}$ signaling pathway involves $\mathrm{I} \kappa \mathrm{B}$ kinase activation and $\mathrm{I} \kappa \mathrm{B}$ phosphorylation, thereby inducing the expression of downstream genes associated with this particular pathway $(32,33)$. The most common NF- $\kappa \mathrm{B}$ dimers are composed of p50 and RelA $(34,35)$. In addition, the $\mathrm{NF}-\kappa \mathrm{B}$ signaling pathway has been reported to contribute to the progression of cervical cancer $(36,37)$. In the present study, the $N F-\kappa B$ signaling pathway was activated in cervical cancer cells; however, overexpression of TAGLN2 inhibited the expression of RelA and $\mathrm{p} 50$, but promoted the expression of $\mathrm{I} \kappa \mathrm{B}$, thus indicating that TAGLN2 suppressed the NF- $\kappa$ B signaling pathway.

In the present study, the tumor-suppressing role of TAGLN2 was proposed in cervical cancer cells. Consistently, the cancer-inhibiting function of TAGLN2 has been reported (11); however, in another study, downregulation of TAGLN2 inhibited the migration and invasion in breast cancer cells (38). Additionally, a previous study revealed that in cervical cancer, suppression of TAGLN2 inhibited cell migration, MMP secretion and cell growth in vitro, and reduced tumor size in vivo (39); variations in observations may be due to differing cellular conditions. Furthermore, the small sample size of the current study may affect the accuracy of the data. Therefore, the role of TAGLN2 requires further in vivo and in vitro investigation, as well as the use of larger sample sizes. In addition, a limitation of the present study was that only TAGLN2 overexpression was performed to explore the role of TAGLN2 in cervical cancer. Therefore, experiments using TAGLN2 knockdown may also provide important insight.

In conclusion, the expression levels of TAGLN2 were lower in cervical cancer tissues, which was associated with the clinical features of patients with CRC. In HeLa cervical cancer cells (HPV-18-positive), TAGLN2 was revealed to inhibit cell viability, migration and invasion in the present study. The biological mechanism underlying the effects of TAGLN2 may be associated with the EMT process and the regulation of EMT-associated factors, as well as the NF- $\kappa \mathrm{B}$ signaling pathway. The present study proposed a novel target gene for the diagnosis, treatment and prognosis of cervical cancer; however, future investigations regarding the regulation of TAGLN2 in cervical cancer in vivo are required.

\section{Acknowledgements}

Not applicable.

\section{Funding}

No funding was received.

\section{Availability of data and materials}

The datasets used and/or analyzed during the current study are available from the corresponding author on reasonable request.

\section{Authors' contributions}

QZ and XD designed the study. QZ, XJ and WY performed the experiments. QZ, XJ and WY performed data analysis. QZ wrote the manuscript. QZ and XD contributed to manuscript revisions, and all authors reviewed the manuscript. All authors read and approved the final manuscript.

\section{Ethics approval and consent to participate}

All sample collections were approved by an institutional review board of the Ethics Committee of The First Affiliated Hospital of Zhejiang Chinese Medical University (Hangzhou, China), and informed consent from each patient was obtained.

\section{Patient consent for publication}

Informed consent was obtained from all participants for the publication of their data.

\section{Competing interests}

The authors declare that they have no competing interests.

\section{References}

1. Wang $\mathrm{J}$ and Yue $\mathrm{X}$ : Role and importance of the expression of transcription factor FOXC2 in cervical cancer. Oncol Lett 14: 6627-6631, 2017

2. Tsakogiannis D, Moschonas GD, Bella E, Kyriakopoulou Z, Amoutzias GD, Dimitriou TG, Kottaridi C and Markoulatos P: Association of p16 (CDKN2A) polymorphisms with the development of HPV16-related precancerous lesions and cervical cancer in the Greek population. J Med Virol 90: 965-971, 2017.

3. Senapati R, Nayak B, Kar SK and Dwibedi B: HPV genotypes co-infections associated with cervical carcinoma: Special focus on phylogenetically related and non-vaccine targeted genotypes. PLoS One 12: e0187844, 2017.

4. Gilmore TD: Introduction to NF-kappaB: players, pathways, perspectives. Oncogene 25: 6680-6684, 2006.

5. Perkins ND: Integrating cell-signalling pathways with NF-kappaB and IKK function. Nat Rev Mol Cell Biol 8: 49-62, 2007.

6. Epinat JC and Gilmore TD: Diverse agents act at multiple levels to inhibit the Rel/NF-kappaB signal transduction pathway. Oncogene 18: 6896-6909, 1999.

7. Gilmore TD: The Rel/NF-kappaB signal transduction pathway: Introduction. Oncogene 18: 6842-6844, 1999.

8. Assinder SJ, Stanton JA and Prasad PD: Transgelin: An actin-binding protein and tumour suppressor. Int J Biochem Cell Biol 41: 482-486, 2009.

9. Takahashi K and Nadal-Ginard B: Molecular cloning and sequence analysis of smooth muscle calponin. J Biol Chem 266: 13284-13288, 1991.

10. Prasad PD, Stanton JA and Assinder SJ: Expression of the actin-associated protein transgelin (SM22) is decreased in prostate cancer. Cell Tissue Res 339: 337-347, 2010.

11. Leung WK, Ching AK, Chan AW, Poon TC, Mian H, Wong AS To KF and Wong N: A novel interplay between oncogenic PFTK1 protein kinase and tumor suppressor TAGLN2 in the control of liver cancer cell motility. Oncogene 30: 4464-4475, 2011.

12. Leinweber BD, Leavis PC, GrabarekZ, Wang CL and Morgan KG: Extracellular regulated kinase (ERK) interaction with actin and the calponin homology $(\mathrm{CH})$ domain of actin-binding proteins. Biochem J 344: 117-123, 1999.

13. Nair RR, Solway J and Boyd DD: Expression cloning identifies transgelin (SM22) as a novel repressor of 92-kDa type IV collagenase (MMP-9) expression. J Biol Chem 281: 26424-26436, 2006.

14. Nohata N, Sone Y, Hanazawa T, Fuse M, Kikkawa N, Yoshino H, Chiyomaru T, Kawakami K, Enokida H, Nakagawa M, et al: miR-1 as a tumor suppressive microRNA targeting TAGLN2 in head and neck squamous cell carcinoma. Oncotarget 2: 29-42, 2011. 
15. Kawakami K, Enokida H, Chiyomaru T, Tatarano S, Yoshino H, Kagara I, Gotanda T, Tachiwada T, Nishiyama K, Nohata N, et al: The functional significance of miR-1 and miR-133a in renal cell carcinoma. Eur J Cancer 48: 827-836, 2012.

16. Rhodes LV, Nitschke AM, Segar HC, Martin EC, Driver JL, Elliott S, Nam SY, Li M, Nephew KP, Burow ME and Collins-Burow BM: The histone deacetylase inhibitor trichostatin A alters microRNA expression profiles in apoptosis-resistant breast cancer cells. Oncol Rep 27: 10-16, 2012.

17. American College of Obstetricians and Gynecologists: ACOG practice bulletin. Diagnosis and treatment of cervical carcinomas. Number 35, May 2002. American College of Obstetricians and Gynecologists. Int J Gynecol Obstet 78: 79-91, 2002.

18. Livak KJ and Schmittgen TD: Analysis of relative gene expression data using real-time quantitative PCR and the 2(-Delta Delta C(T)) method. Methods 25: 402-408, 2001.

19. Waggoner SE: Cervical cancer. Lancet 361: 2217-2225, 2003.

20. Stehman FB, Rose PG, Greer BE, Roy M, Plante M, Penalver M, Jhingran A, Eifel P, Montz F and Wharton JT: Innovations in the treatment of invasive cervical cancer. Cancer 98: 2052-2063, 2003.

21. Munagala R, Donà MG, Rai SN, Jenson AB, Bala N, Ghim SJ and Gupta RC: Significance of multiple HPV infection in cervical cancer patients and its impact on treatment response. Int J Oncol 34: 263-271, 2009.

22. Mani SA, Guo W, Liao MJ, Eaton EN, Ayyanan A, Zhou AY, Brooks M, Reinhard F, Zhang CC, Shipitsin M, et al: The epithelial-mesenchymal transition generates cells with properties of stem cells. Cell 133: 704-715, 2008

23. Radisky DC: Epithelial-mesenchymal transition. Cancer Res 118 4325-4326, 2005.

24. Yang $J$ and Weinberg RA: Epithelial-mesenchymal transition: At the crossroads of development and tumor metastasis. Dev Cell 14: 818-829, 2008.

25. Korpal M, Lee ES, Hu G and Kang Y: The miR-200 family inhibits epithelial-mesenchymal transition and cancer cell migration by direct targeting of E-cadherin transcriptional repressors ZEB1 and ZEB2. J Biol Chem 283: 14910-14914, 2008.

26. Hwang TL, Lee LY, Wang CC, Liang Y, Huang SF and Wu CM: Claudin-4 expression is associated with tumor invasion, MMP-2 and MMP-9 expression in gastric cancer. Exp Ther Med 1: 789-797, 2010.

27. Smith MC, Luker KE, Garbow JR, Prior JL, Jackson E, Piwnica-Worms D and Luker GD: CXCR4 regulates growth of both primary and metastatic breast cancer. Cancer Res 64 8604-8612, 2004.
28. Tachibana K, Hirota S, Iizasa H, Yoshida H, Kawabata K, Kataoka Y, Kitamura Y, Matsushima K, Yoshida N, Nishikawa S, et al: The chemokine receptor CXCR4 is essential for vascularization of the gastrointestinal tract. Nature 393: 591-594, 1998.

29. Baldwin AS Jr: The NF-kappa B and I kappa B proteins: New discoveries and insights. Annu Rev Immunol 14: 649-683, 1996.

30. Vallabhapurapu S and Karin M: Regulation and function of NF-kappaB transcription factors in the immune system. Annu Rev Immunol 27: 693-733, 2009.

31. Lenardo MJ and Baltimore D: NF-kappaB: A pleiotropic mediator of inducible and tissue-specific gene control. Cell 58: 227-229, 1989.

32. Thompson JE, Phillips RJ, Erdjument-Bromage H, Tempst $\mathrm{P}$ and Ghosh S: I kappa B-beta regulates the persistent response in a biphasic activation of NF-kappa B. Cell 80: 573-582, 1995.

33. DiDonato JA, Hayakawa M, Rothwarf DM, Zandi E and Karin M: A cytokine-responsive IkappaB kinase that activates the transcription factor NF-kappaB. Nature 388: 548-554, 1997.

34. Greten FR, Arkan MC, Bollrath J, Hsu LC, Goode J, Miething C, Göktuna SI, Neuenhahn M, Fierer J, Paxian S, et al: NF-kappaB is a negative regulator of IL-1beta secretion as revealed by genetic and pharmacological inhibition of IKKbeta. Cell 130: 918-931, 2007.

35. Rahighi S, Ikeda F, Kawasaki M, Akutsu M, Suzuki N, Kato R, Kensche T, Uejima T, Bloor S, Komander D, et al: Specific recognition of linear ubiquitin chains by NEMO is important for NF-kappaB activation. Cell 136: 1098-1109, 2009.

36. Ramdass B, Maliekal TT, Lakshmi S, Rehman M, Rema P, Nair P, Mukherjee G, Reddy BK, Krishna S and Radhakrishna Pillai M: Coexpression of Notch1 and NF-kappaB signaling pathway components in human cervical cancer progression. Gynecol Oncol 104: 352-361, 2007.

37. Ma XF, Zhang J, Shuai HL, Guan BZ, Luo X and Yan RL: $\mathrm{IKK} \beta / \mathrm{NF}-\kappa \mathrm{B}$ mediated the low doses of bisphenol A induced migration of cervical cancer cells. Arch Biochem Biophys 573: $52-58,2015$

38. Zheng X, Chen S, Yang Q, Cai J, Zhang W, You H, Xing J and Dong Y: Salvianolic acid A reverses the paclitaxel resistance and inhibits the migration and invasion abilities of human breast cancer cells by inactivating transgelin 2. Cancer Biol Ther 16: 1407-1414, 2015.

39. Yakabe K, Murakami A, Kajimura T, Nishimoto Y, Sueoka K, Sato S, Nawata $S$ and Sugino N: Functional significance of transgelin-2 in uterine cervical squamous cell carcinoma. J Obstet Gynaecol Res 42: 566-572, 2016. 